\title{
Initial Characterization of Interstellar Comet 2I/2019 Q4 (Borisov)
}

\author{
David Jewitt ${ }^{1,2}$ and Jane Luu ${ }^{3}$ \\ ${ }^{1}$ Department of Earth, Planetary and Space Sciences, UCLA, 595 Charles Young Drive East, Los Angeles, CA 90095-1567, USA; jewitt@ucla.edu \\ ${ }^{2}$ Department of Physics and Astronomy, UCLA, 430 Portola Plaza, Box 951547, Los Angeles, CA 90095-1547, USA \\ 3 Centre for Earth Evolution and Dynamics, University of Oslo, Postboks 1028 Blindern, NO-0315 Oslo, Norway \\ Received 2019 October 4; revised 2019 October 24; accepted 2019 October 30; published 2019 November 22
}

\begin{abstract}
We present initial observations of the interstellar body 2I/(2019 Q4) Borisov taken to determine its nature prior to the perihelion in 2019 December. Images from the Nordic Optical Telescope show a prominent, morphologically stable dust coma and tail. The dust cross-section within $15,000 \mathrm{~km}$ of the nucleus averages $130 \mathrm{~km}^{2}$ (assuming geometric albedo 0.1) and increases by about $1 \%$ per day. If sustained, this rate indicates that the comet has been active for $\sim 100$ days prior to the observations. Cometary activity thus started in 2019 June, at which time C/ Borisov was at $\sim 4.5$ au from the Sun, a typical distance for the onset of water ice sublimation in comets. The dust optical colors, $B-V=0.80 \pm 0.05, V-R=0.47 \pm 0.03$ and $R-I=0.49 \pm 0.05$, are identical to those of a sample of (solar system) long-period comets. The colors are similar to those of 1I/(2017 U1) 'Oumuamua, indicating a lack of the ultrared matter that is common in the Kuiper Belt, on both interstellar objects. The effective size of the dust particles is estimated as $\bar{a}=100 \mu \mathrm{m}$, based on the length of the dust tail and the 100 day lifetime. With this size, the ejected dust mass is of order $1.3 \times 10^{7} \mathrm{~kg}$ and the current dust mass loss rate $\sim 2 \mathrm{~kg} \mathrm{~s}{ }^{-1}$. We set an upper limit to the nucleus radius using photometry at $r_{n} \leqslant 3.8 \mathrm{~km}$ (again for albedo 0.1 ) and we use a statistical argument to show that the nucleus must be much smaller, likely a few hundred meters in radius.
\end{abstract}

Unified Astronomy Thesaurus concepts: Comets (280)

\section{Introduction}

Object 2I/(2019 Q4) Borisov (hereafter "Q4") was discovered by G. Borisov of the Moscow State University on UT 2019 August 30 and publicly announced on September 11 (Borisov 2019). It is the second known interstellar object in the solar system, after 1I/(2017 U1) 'Oumuamua, and the first interstellar comet. Discovered at only $38^{\circ}$ solar elongation, Q4 represents both a triumph of small-telescope astronomy and a challenge for observers using large telescopes, few of which can be operated at such small angles from the Sun. In this report, we describe initial observations from the $2.56 \mathrm{~m}$ diameter Nordic Optical Telescope (NOT), designed to provide a first characterization of the object.

\section{Observations}

The small elongation of Q4 and the requirement that optical observations be taken against a dark sky forced us to observe at low elevations immediately before sunrise. The NOT, located at $2400 \mathrm{~m}$ altitude in the Canary Islands, can take useful data at elevations as small as 6.4 (airmass 9). On UT 2019 September 13 and 14, we employed the $1024 \times 1024$ pixel StanCam, with 0 "' 176 pixels giving a 3! $0 \times 3$ ! 0 field of view. On September $15,18,26$ and October 4 we used the $2048 \times 2048$ pixel ALFOSC camera, which has a $6.5 \times 6.5$ field of view with 0 ". 214 pixels. The telescope was tracked non-sidereally to follow the motion of the comet (approximate rates $60^{\prime \prime} \mathrm{hr}^{-1}$ east and $50^{\prime \prime} \mathrm{hr}^{-1}$ south). We began observing at airmasses as high as $\sim 8$ in observing windows that were soon truncated by morning twilight. Furthermore, owing to the urgency of the observations our initial data were taken in the presence of scattered light from the full Moon. Both the airmass and the Moon phase improved, however, and the later observations were possible at more modest airmasses, $\sim 2-3$, and against a dark sky. A journal of observations is given in Table 1.
We used broadband BVRI filters approximating the Bessell (1995) system to measure Q4. The central wavelengths, $\lambda_{c}$, and FWHM of the ALFOSC filters in the form Filter $\left(\lambda_{c}, \mathrm{FWHM}\right)$ are $B(4400,1000), V(5300,800), R(6500,1300)$, and $i_{\text {int }}(7970$, $1570)$, with all wavelengths expressed in $\AA$. The StanCam $V$ filter is slightly different, $V(5430,1030)$. Flat fields were constructed after debiasing using nightly images of the illuminated interior of the observatory dome. The data were photometrically calibrated both with respect to field stars in the Sloan data release 14 (DR14; Blanton et al. 2017) and through observations of Landolt (1992) photometric standard stars. Use of the Sloan field stars entails no airmass correction, but necessitates a transformation from the Sloan magnitude system to Bessel magnitudes using the relations given by Jordi et al. (2006). To use the Landolt stars, which were necessarily observed at airmasses different from those of the comet, we measured and applied extinction coefficients of $k_{B}=0.23$, $k_{V}=0.14$, and $k_{R}=0.11 \mathrm{mag}$ per airmass. We did not measure $k_{I}$ but instead assume $k_{I}=0.06$ magnitudes per airmass.

\section{Discussion}

Morphology: Except for differences in the sensitivity to low surface brightness material caused by nightly variations in the sky brightness, the appearance of Q4 did not change between the different nights of observation (Table 1). Figure 1 shows a representative $R$-band image composite from UT 2019 September 26 (fraction of Moon illuminated $\sim 10 \%$ ) formed by aligning and combining eight images each of $180 \mathrm{~s}$ duration. The left panel shows the unadorned image, the middle panel adds contours to highlight the tail, and the right panel has been smoothed by convolution with a Gaussian function having $\mathrm{FWHM}=1^{\prime \prime}$ to emphasize faint structure. Vectors $-V$ and $-\odot$ show the projected negative orbital velocity and the projected anti-solar direction (see also Table 1). The comet is 
Table 1

Observing Geometry

\begin{tabular}{|c|c|c|c|c|c|c|c|c|c|}
\hline UT Date and Time & Airmass $^{\mathrm{a}}$ & Day of Year (DOY) & $\Delta T_{p}^{\mathrm{c}}$ & $r_{H}^{\mathrm{d}}$ & $\overline{\Delta^{\mathrm{e}}}$ & $\alpha^{\mathrm{f}}$ & $\theta_{\odot}^{\mathrm{g}}$ & $\theta_{-\mathrm{V}}^{\mathrm{h}}$ & $\delta_{\oplus}{ }^{\mathrm{i}}$ \\
\hline 2019 Sep 13 04:12-06:02 & $6.49-1.94$ & 256 & -86 & 2.767 & 3.407 & 14.5 & 298.6 & 326.8 & -7.9 \\
\hline 2019 Sep 14 04:22-06:05 & $5.18-1.90$ & 257 & -85 & 2.752 & 3.385 & 14.7 & 298.3 & 326.9 & -8.1 \\
\hline 2019 Sep 15 04:40-06:08 & $3.94-1.85$ & 258 & -84 & 2.737 & 3.363 & 14.9 & 298.0 & 327.0 & -8.3 \\
\hline 2019 Sep 18 04:01-05:17 & $7.16-2.54$ & 261 & -81 & 2.693 & 3.297 & 15.5 & 297.3 & 327.4 & -8.9 \\
\hline 2019 Sep 26 05:03-06:00 & $2.59-1.76$ & 269 & -73 & 2.580 & 3.123 & 17.1 & 295.5 & 328.4 & -10.4 \\
\hline 2019 Oct 4 05:25-05:49 & $2.02-1.75$ & 277 & -65 & 2.470 & 2.950 & 18.7 & 294.0 & 329.3 & -12.1 \\
\hline
\end{tabular}

Notes.

${ }^{a}$ Airmass at the start and end time of observation.

${ }^{\text {b }}$ UT 2019 January $1=1$.

${ }^{\mathrm{c}}$ Number of days from perihelion (UT 2019 December 8=DOY 342). Negative numbers indicate pre-perihelion observations.

d Heliocentric distance, in au.

e Geocentric distance, in au.

${ }^{\mathrm{f}}$ Phase angle, in degrees.

${ }^{g}$ Position angle of the projected anti-Solar direction, in degrees.

${ }^{\mathrm{h}}$ Position angle of the projected negative heliocentric velocity vector, in degrees.

${ }^{\mathrm{i}}$ Angle of Earth above the orbital plane, in degrees.

clearly non-stellar, and shows an extensive dust tail to the northwest, approximately bounded by the projected orbit and anti-solar vectors, as is a characteristic of dust tails. The visible portion of the tail is limited to about $60^{\prime \prime}$ in length by sky noise and field structure from trailed field stars and galaxies. This corresponds to a sky-plane length $L=1.4 \times 10^{8} \mathrm{~m}$. If the tail is in fact anti-solar, then its true length is given by $L_{0}=L / \sin (\alpha)$ which, with $\alpha=17^{\circ}$ (Table 1 ), gives $L_{0}=4.8 \times 10^{8} \mathrm{~m}$.

The surface brightness profile of Q4 is compared with that of a field star in Figure 2. Both profiles were computed by averaging the signal within a set of concentric circular apertures centered on the optocenters of each object. Sky subtraction was determined from the median signal within a concentric annulus having inner and outer radii 200 pixels (42".8) and 107!"0, respectively. We experimented with the radii of the sky annulus, finding no significant effect on the profile over the region measured. The figure shows the extended nature of Q4. The central region of the profile is strongly affected by the point-spread function of the data and we do not attempt to model it here. We fitted a power law to the surface brightness profile over the radius range $5^{\prime \prime}-22^{\prime \prime}$, finding $\Sigma(\theta) \propto \theta^{m}$, where $\Sigma(\theta)$ is the normalized surface brightness at radius $\theta$ and index $m=-1.85 \pm 0.02$. This value is steep compared to $m=-1$, as expected of an isotropic coma in steady state, and also steeper than the value $m=-3 / 2$ resulting from the action of radiation pressure on an otherwise steady-state coma (Jewitt \& Meech 1987). Interpretation of this profile is deferred to the acquisition of more data on Q4 as it rounds perihelion. We merely note that steeper profiles can result from fading grains or, more plausibly, a dust production rate rising with time.

Photometry: We measured the brightness of Q4 in each image within circular apertures having projected radii $7500 \mathrm{~km}$ and $15,000 \mathrm{~km}$ (roughly $3^{\prime \prime}$ and $6^{\prime \prime}$, respectively, although varying with the geocentric distance to Q4). Sky subtraction was obtained using the median level within a contiguous, concentric annulus of width 10". 7. The difference between Q4 and the field stars was used to negate the effects of changing atmospheric extinction. After this correction, no convincing photometric variability was detected within each night. As an example, Figure 3 shows $V$-filter photometry from a $\sim 2 \mathrm{hr}$ timespan on UT 2019 September 15, during which time the comet rose from airmass 6.5 to 1.9 . Representative error bars of \pm 0.08 mag are included. The mean and standard error on the mean of the plotted data are $V=18.02 \pm 0.03 \quad(n=20$ measurements), with no evidence for a systematic trend in the magnitude over this period. Deviations on timescales $\sim 30$ minutes are likely related to seeing and guiding fluctuations given the high airmass of these observations, especially near the beginning of the observing window (UT 4-5 hr). This photometric invariance is a natural result of coma dilution within the photometric aperture (Jewitt 1991). This occurs when the timescale for particles to cross the aperture, $\tau_{\text {cross }}$, is comparable to or longer than the timescale for variation of the source. For example, small dust particles well-coupled to the outflowing gas would leave the nucleus with a speed comparable to the speed of sound in gas at the local blackbody temperature $\left(V_{s} \sim 0.4 \mathrm{~km} \mathrm{~s}^{-1}\right.$ for gaseous $\mathrm{H}_{2} \mathrm{O}$ with $T_{\mathrm{BB}}=168$ $\mathrm{K}$ at $2.74 \mathrm{au}$ ). Then, with aperture radius $\ell=15,000 \mathrm{~km}$, we find $\tau_{\text {cross }}=\ell / V_{s} \sim 3.8 \times 10^{4} \mathrm{~s}$ (about $10 \mathrm{hr}$ ), and larger (slower) particles will take a longer time. Photometric variations on timescales $\lesssim 10 \mathrm{hr}$ must necessarily be damped by aperture-averaging. For comparison, 1I/(2017 U1) 'Oumuamua was devoid of coma and showed an extreme lightcurve with period $\sim 8 \mathrm{hr}$ and a range $\sim 2.5$ magnitudes (Meech et al. 2017), indicating an axis ratio of 5:1 (Bannister et al. 2017; Drahus et al. 2018). Coma dilution would render such a lightcurve in Q4 invisible.

In order to search for variations on longer timescales, we compared the averaged photometry from each night of observation. The observing geometry changes significantly between nights (Table 1) so we compared absolute magnitudes, $H$, computed using

$$
H=V-5 \log _{10}\left(r_{h} \Delta\right)-f(\alpha)
$$

where $V$ is the apparent magnitude and $f(\alpha)$ is the phase function. The backscattering phase functions of comets are in general poorly known and that of Q4 is completely unmeasured. We used $f(\alpha)=0.04 \alpha$, which gives the ratio of scattered fluxes at $0^{\circ}$ phase and $15^{\circ}$ phase as $B=1.7$, comparable to values $B \sim 2$ measured in $67 \mathrm{P} /$ ChuryumovGerasimenko (Bertini et al. 2019). 

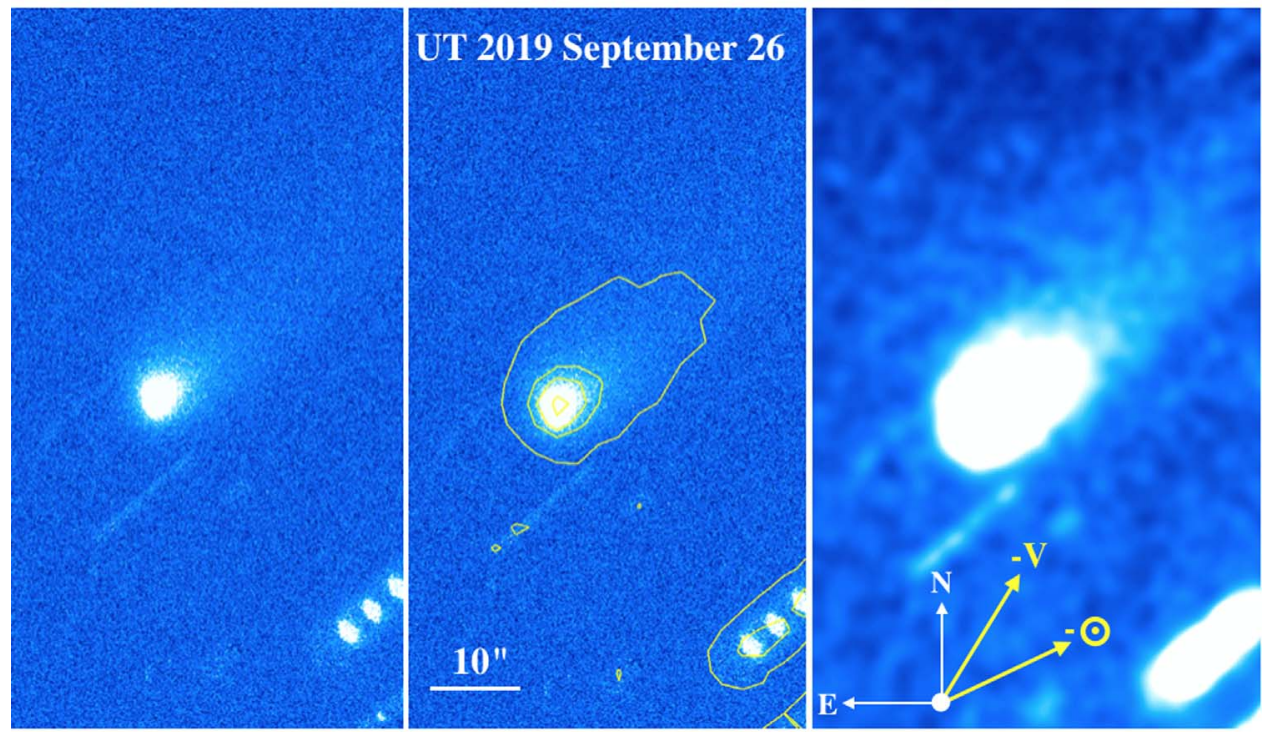

Figure 1. Composite of eight images of Q4, each of $180 \mathrm{~s}$ duration, taken through the R filter on UT 2019 September 26. The three panels show (left) the raw composite, (middle) added contours to highlight the coma, and (right) a spatially smoothed version, to show the faintest dust. White arrows show the directions of north and east, while yellow arrows marked $-\odot$ and $-V$ show the projected anti-solar and anti-velocity vectors. A $10^{\prime \prime}\left(2.3 \times 10^{4} \mathrm{~km}\right)$ scale bar is shown.

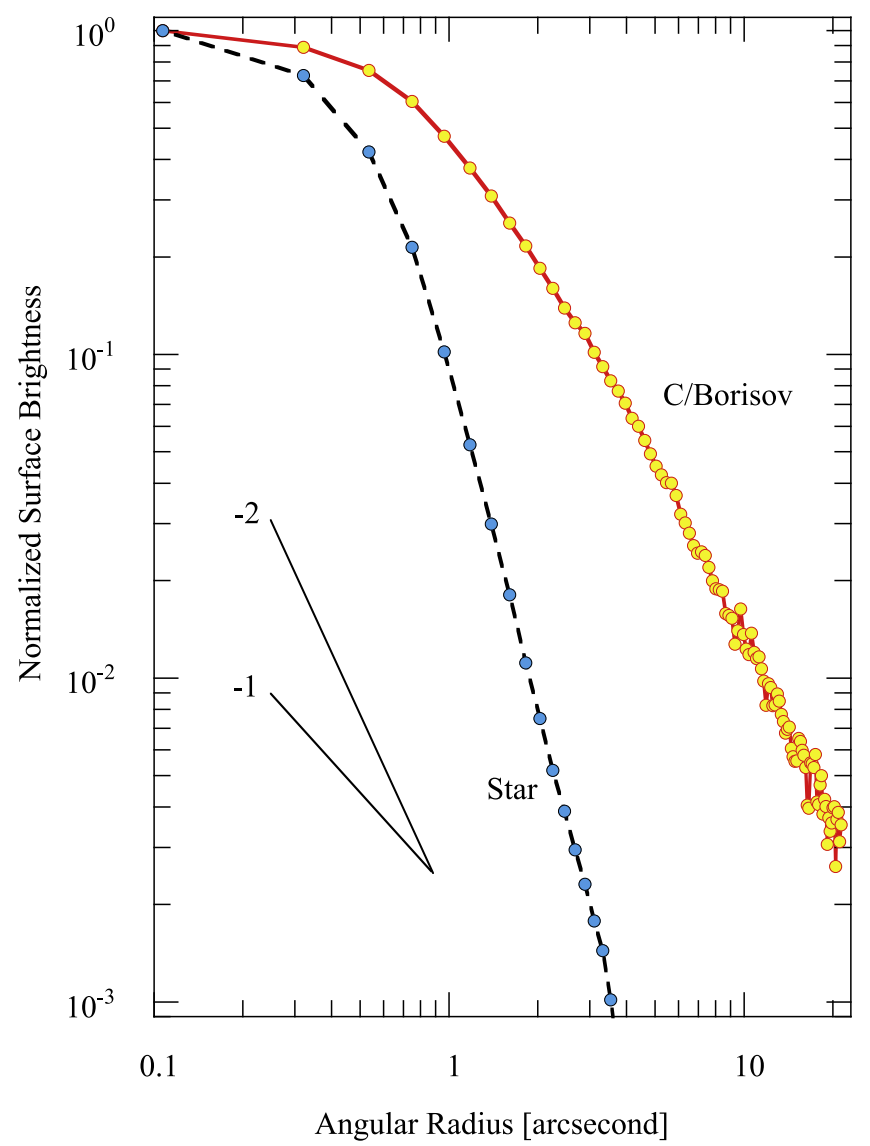

Figure 2. Surface brightness profiles of Q4 and a field star from UT 2019 September 26, in the $R$ filter. Straight lines show surface brightness gradients $m=-1$ and $m=-2$, as marked.

The absolute magnitude is further related to the effective scattering cross-section, $C_{e}\left[\mathrm{~km}^{2}\right]$, by

$$
C_{e}=\frac{1.5 \times 10^{6}}{p_{V}} 10^{-0.4 H}
$$

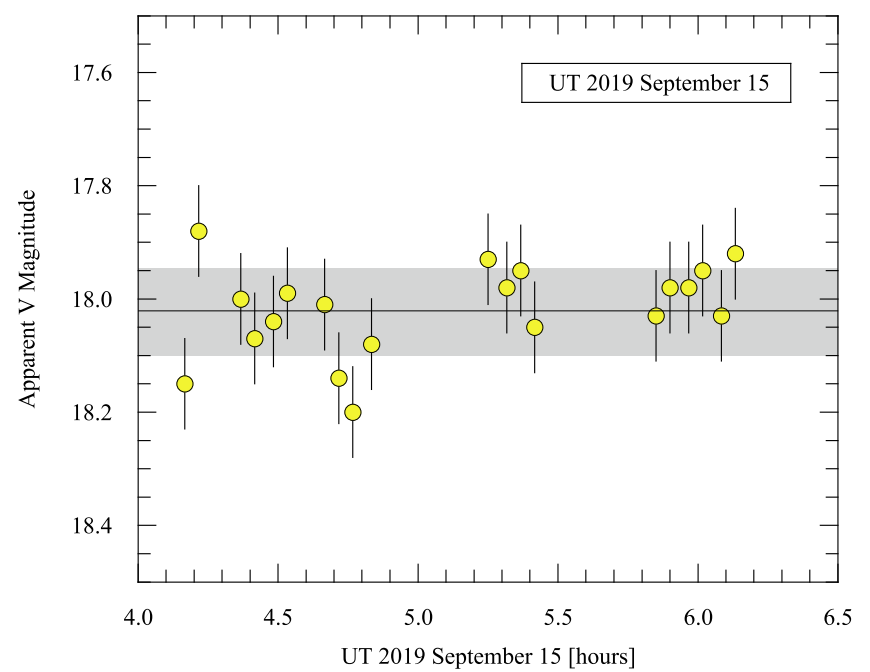

Figure 3. Lightcurve on UT 2019 September 15 measured in the $V$ filter within a projected aperture $15,000 \mathrm{~km}$ in radius. The horizontal line shows the mean value, $V=18.02$.

where $p_{V}$ is the geometric albedo. We assume $p_{V}=0.1$, as appropriate for cometary dust (Zubko et al. 2017). A much higher albedo could apply if the coma grains were icy, but the spectroscopic non-detection of water ice absorption bands reported by Yang et al. (2019) suggests that this is not the case. The nightly apparent and absolute magnitudes and the scattering cross-sections are listed in Table 2.

The average cross-section within the $15,000 \mathrm{~km}$ aperture is $C_{e} \sim 130 \mathrm{~km}^{2}$, with a slight dependence on time. Figure 4 shows $C_{e}$ versus time together with a weighted linear, leastsquares fit to the data having best-fit gradient $d C_{e} / d t=1.26 \pm 0.25 \mathrm{~km}^{2}$ day $^{-1}$ (i.e., about $1 \% \mathrm{day}^{-1}$ ). The magnitude of $d C_{e} / d t$ is influenced by the adopted phase function but, because the range of phase angles in our data is small (Table 1) the effect is modest. Phase functions in the range $f(\alpha)=0.03-0.05$ mag degree ${ }^{-1}$ change the gradient by an amount smaller than the statistical error. Extrapolation of the 
Table 2

Photometry with Fixed Linear Apertures

\begin{tabular}{lccc}
\hline \hline UT Date & $V^{\mathrm{a}}$ & $H^{\mathrm{b}}$ & $C_{e}{ }^{\mathrm{c}}$ \\
\hline Sep 13 & $18.12 \pm 0.05$ & 12.67 & $128 \pm 6$ \\
Sep 14 & $18.24 \pm 0.13$ & 12.81 & $113 \pm 16$ \\
Sep 15 & $18.02 \pm 0.03$ & 12.60 & $136 \pm 4$ \\
Sep 18 & $18.02 \pm 0.02$ & 12.66 & $130 \pm 3$ \\
Sep 26 & $17.74 \pm 0.02$ & 12.53 & $146 \pm 3$ \\
Oct 4 & $17.55 \pm 0.03$ & 12.49 & $151 \pm 5$ \\
\hline
\end{tabular}

Notes.

a Apparent $V$-band magnitude within $15,000 \mathrm{~km}$ radius projected aperture.

b Absolute magnitude computed from Equation (1). The statistical uncertainty on $H$ is the same as on $V$ but with an additional systematic uncertainty owing to the unknown phase function.

${ }^{\mathrm{c}}$ Cross-section in $\mathrm{km}^{2}$ computed from $H$ using Equation (2) with $p_{V}=0.1$.

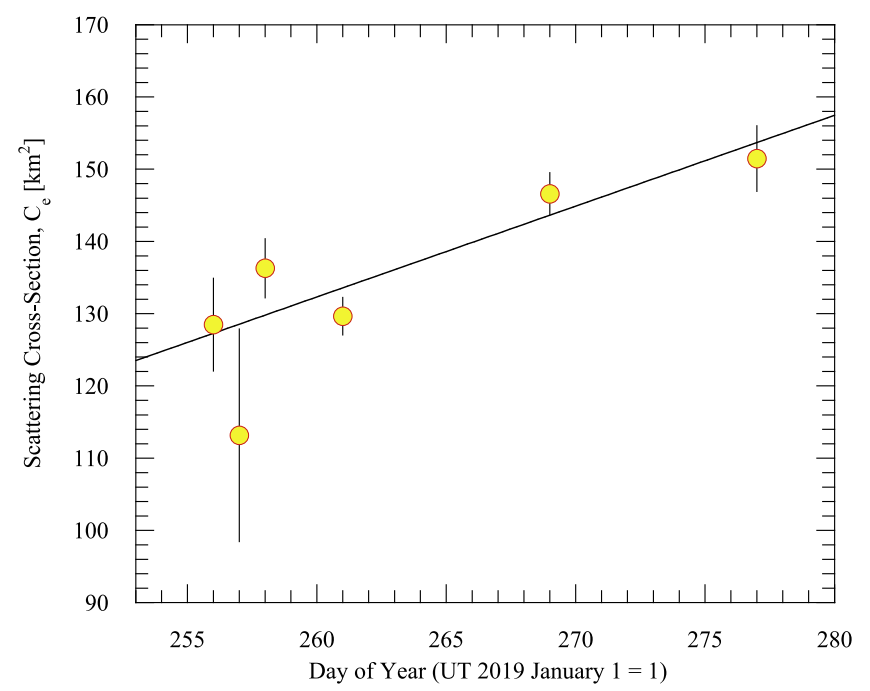

Figure 4. Scattering cross-section within a circular aperture $1.5 \times 10^{4} \mathrm{~km}$ in radius, as a function of time, expressed as Day of Year (DOY=1 on UT 2019 January 1). The line shows a linear, least-squares fit having gradient $1.26 \pm 0.25 \mathrm{~km}^{2} \mathrm{day}^{-1}$. Data from Table 2 .

data gives $C_{e}=0 \mathrm{~km}^{2}$ about 100 days before the first observation on September 13, corresponding to DOY 156 (UT 2019 June 5, when Q4 was at $r_{H}=4.5 \mathrm{au}$ ). We possess no proof that such an extrapolation is justified, but it is noteworthy that the inferred turn-on distance matches the $r_{H} \sim 4-5 \mathrm{au}$ critical distance at which water ice sublimation begins in comets.

The optical cross-section is almost entirely carried by dust and we can use it only to derive upper limits to the size of the nucleus. A crude upper limit is given by $r_{n}=\left(C_{e} / \pi\right)^{1 / 2}$ with $C_{e}=130 \mathrm{~km}^{2}$. Substituting gives $r_{n} \leqslant 6.4 \mathrm{~km}$, again assuming $p_{V}=0.1$. We sought a stronger limit using photometry from smaller, less dust-contaminated apertures. By experimentation, we found that photometry within apertures of radius $<2$ !" 1 (10 ALFOSC pixels, or about $4800 \mathrm{~km}$ at the distance of Q4) was unduly sensitive to variations in the image point-spread function caused by a combination of atmospheric turbulence and telescope tracking. Photometry within a 2 !! 1 radius aperture on UT 2019 September 26, with background subtraction of the coma from a contiguous annulus of outer radius 4 ". 2 , gives $V=19.03 \pm 0.03$. This corresponds through Equation (1) to $H=13.82$ and through Equation (2) to $C_{e}=45 \mathrm{~km}^{2}$ and $r_{n}<3.8 \mathrm{~km}$. We emphasize that this is still a strong upper limit to $r_{n}$ because of dust contamination in the photometry aperture. More stringent observational constraints on the nucleus await the acquisition of high angular resolution data and/or the cessation of activity as Q4 recedes from the Sun, post-perihelion.

The mass of dust, $M$, and its cross-section, $C_{e}$ are related by $M \sim 4 \rho \bar{a} C_{e} / 3$, where $\bar{a}$ is the mean dust particle radius and $\rho$ is the particle density. We take $\rho=10^{3} \mathrm{~kg} \mathrm{~m}^{-3}$ as the nominal density. The mean particle radius is obtained from the tail length, mentioned above as $L_{0}=4.8 \times 10^{8} \mathrm{~m}$. We assume that these particles were ejected from the nucleus a time $t \sim 100$ days $\left(\sim 9 \times 10^{6} \mathrm{~s}\right)$ ago and that their deflection into a tail is the result of solar radiation pressure acceleration. We write the radiation pressure acceleration as $\beta g_{\odot}(1) r_{H}^{-2}$, where $\beta$ is a (dimensionless) function of the particle properties and $g_{\odot}(1)=0.006 \mathrm{~m} \mathrm{~s}^{-2}$ is the gravitational acceleration toward the Sun at $r_{H}=1 \mathrm{au}$. Neglecting the variation of $r_{H}$ over the 100 day flight time, and neglecting their initial velocity, we estimate $\beta$ from

$$
\beta \sim \frac{2 L_{0} r_{H}^{2}}{g_{\odot} t^{2}}
$$

with $r_{H}$ expressed in au. We set $r_{H}=2.580$ au (September 26) to find $\beta \sim 0.01$. In dielectric spheres, $\beta$ is approximately equal to the inverse particle radius expressed in microns (Bohren $\&$ Huffman 1983). Therefore, $\beta \sim 0.01$ implies effective particle size $\bar{a}=100 \mu \mathrm{m}$. Strictly, this estimate applies to particles displaced to the end of the visible tail and particles closer to the nucleus could be much larger. We preliminarily take $\bar{a}=100 \mu \mathrm{m}$ as the nominal particle radius, pending more accurate determinations.

Substituting, we find dust mass $M \sim 1.3 \times 10^{7} \mathrm{~kg}$. The rate of production of dust is $d M / d t=4 \rho \bar{a}\left(d C_{e} / d t\right) / 3 \sim 2 \times 10^{5} \mathrm{~kg} \mathrm{day}^{-1}\left(2 \mathrm{~kg} \mathrm{~s}^{-1}\right)$. For comparison, the only other currently available constraint on the mass loss rate is from a reported $\mathrm{CN}$ production rate $Q_{\mathrm{CN}} \sim(3.7 \pm 0.4) \times 10^{24} \mathrm{~s}^{-1}\left(0.2 \mathrm{~kg} \mathrm{~s}^{-1}\right.$; Fitzsimmons et al. 2019). In solar system comets, the ratio of the water to $\mathrm{CN}$ production rates varies about an average value $Q_{\mathrm{H} 2 \mathrm{O}} / Q_{\mathrm{CN}}=360$ (A'Hearn et al. 1995). If this ratio applies to Q4, then we infer a mass loss rate in water of $\dot{M} \sim 60 \mathrm{~kg} \mathrm{~s}^{-1}$, more than an order of magnitude larger than the production rate in dust. The equilibrium mass sublimation flux at 2.7 au for an absorbing water ice surface oriented perpendicular to the Sun direction is $f_{s}=4 \times 10^{-5} \mathrm{~kg} \mathrm{~m}^{-2} \mathrm{~s}^{-1}$, which could be supplied by a patch of area $A=\dot{M} / f_{s} \sim 1.5 \times 10^{6} \mathrm{~m}^{2}$ $\left(1.5 \mathrm{~km}^{2}\right)$. This is equal to the surface area of a sphere of radius $r_{N}=0.35 \mathrm{~km}$ and sets a lower bound to the radius of the nucleus, assuming that nucleus sublimation is the only gas source. By similar arguments, Fitzsimmons et al. (2019) found an upper limit to the radius $r_{n}<8 \mathrm{~km}$ and a preferred range $0.7 \leqslant r_{n} \leqslant 3.3 \mathrm{~km}$.

Colors: The mean colors listed in Table 3 show no dependence on the aperture radius between $3^{\prime \prime}$ and $6^{\prime \prime}$. Our measurement of $V-R$ can be compared with an independent determination, $g-r=0.63 \pm 0.02$ (Guzik et al. 2019, who used a $2^{\prime \prime}$ radius aperture) which, when transformed using the relations of Jordi et al. (2006), gives $V-R=0.49 \pm 0.02$. The agreement is excellent. The table also lists the colors of the Sun, of 1I/(2017 U1) 'Oumuamua (Jewitt et al. 2017) and the mean color of the long-period comets (Jewitt 2015). Figure 5 
Table 3

Color Measurements

\begin{tabular}{|c|c|c|c|c|c|c|}
\hline Object & Date & Aper. $^{\mathrm{a}}$ & $B-V$ & $V-R$ & $R-I$ & $B-R$ \\
\hline Borisov & Sep 15 & 7500 & $\ldots$ & $0.49 \pm 0.04$ & $\ldots$ & $\ldots$ \\
\hline Borisov & Sep 15 & 15,000 & $\ldots$ & $0.53 \pm 0.04$ & $\ldots$ & $\cdots$ \\
\hline Borisov & Sep 26 & 7500 & $0.78 \pm 0.05$ & $0.49 \pm 0.03$ & $0.49 \pm 0.05$ & $1.27 \pm 0.06$ \\
\hline 'Oumuamua ${ }^{\mathrm{b}}$ & $\ldots$ & $\ldots$ & $0.70 \pm 0.06$ & $0.45 \pm 0.05$ & $\ldots$ & $\overline{1.15 \pm 0.08}$ \\
\hline Mean LPC ${ }^{c}$ & $\ldots$ & $\ldots$ & $0.78 \pm 0.02$ & $0.47 \pm 0.02$ & $0.42 \pm 0.03$ & $1.24 \pm 0.02$ \\
\hline
\end{tabular}

Notes.

${ }^{a}$ Aperture radius in $\mathrm{km}$.

${ }^{\mathrm{b}}$ From Jewitt et al. (2017).

${ }^{\mathrm{c}}$ Mean of active long-period comets, 25 observed in $B-V, 24$ in $V-R$ and 7 in $R-I$, from Jewitt (2015).

${ }^{\mathrm{d}}$ From Holmberg et al. (2006).

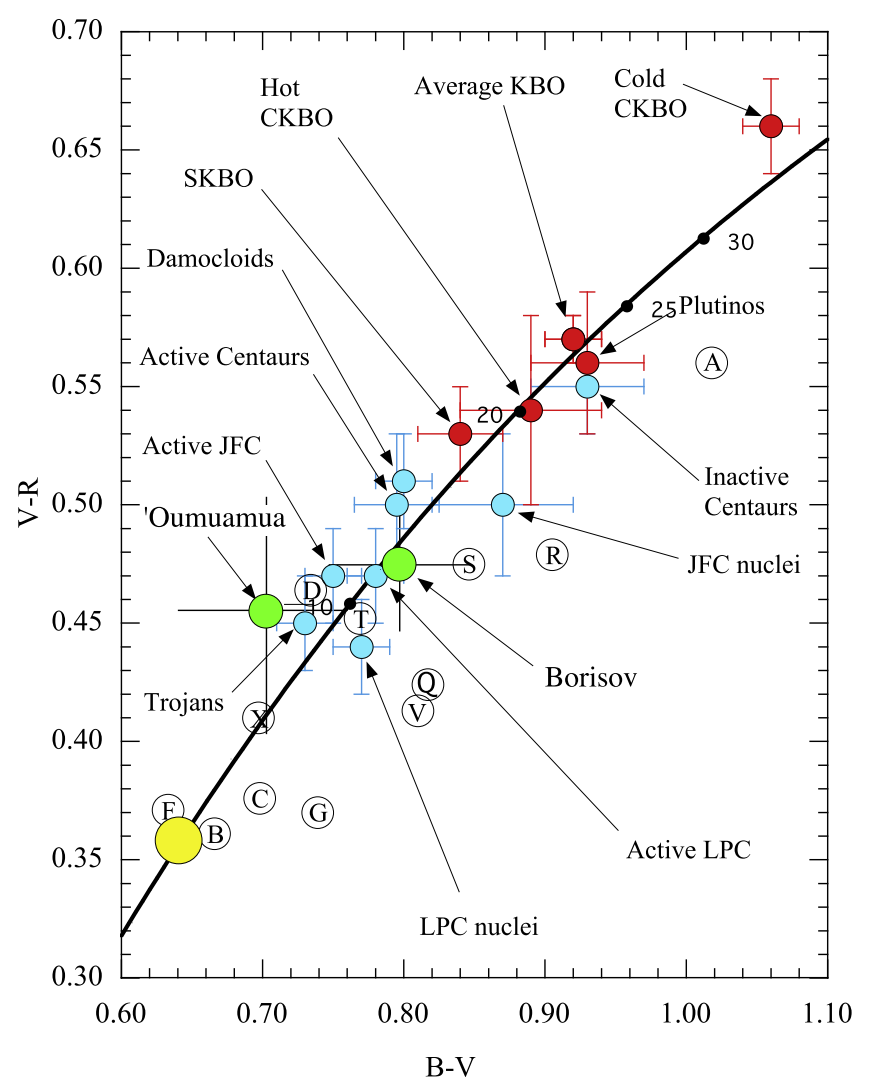

Figure 5. $B-V$ vs. $V-R$ color plane comparing $1 \mathrm{I} /(2017 \mathrm{U} 1)$ 'Oumuamua and Q4 with solar system objects. Red circles indicate sub-types of KBO (hot and cold classical KBOs, 3:2 resonant "Plutinos" and scattered KBOs are distinguished), blue circles indicate the Centaurs and the nuclei and comae of both short- and long-period comets, as labeled, together with the Jupiter Trojans. All data is taken from Jewitt (2015). Letters show the positions of main-belt asteroid spectral classes according to Dandy et al. (2003).

shows the $B-V$ versus $V-R$ color plane for these and other solar system objects, modified from Jewitt (2015). The optical colors of Q4 are redder than the Sun (the reflectivity gradient across the BR region of the spectrum is $S^{\prime} \sim 4 \%$ per $1000 \AA$ ) but closely match the mean colors of (solar system) long-period comets. The colors of Q4 are similar to those of $1 \mathrm{I} /(2017 \mathrm{U} 1)$ 'Oumuamua within the uncertainties of measurement. Neither interstellar object shows evidence for the ultrared matter $\left(S^{\prime} \geqslant 25 \% / 1000 \AA\right)$, which is a prominent feature of many Kuiper Belt objects (KBOs; Jewitt 2002, 2015). The lack of ultrared matter (likely to consist of complex irradiated organics; see Cruikshank et al. 1998; Dalle Ore et al. 2015) mirrors its absence in the solar system at distances $\lesssim 10$ au. As in the active comets of the solar system, we surmise that the particles ejected into the coma and tail of Q4 are derived from beneath a pre-existing ultrared mantle of cosmic-ray irradiated material. The ultrared matter is either thermodynamically unstable in the inner solar system as a result of the elevated temperatures or perhaps ejected or buried by fallback debris (Jewitt 2002).

Statistics of Interstellar Objects: The discovery of Q4 two years after 1I/'Oumuamua exactly matches a published prediction of the discovery rate, namely $S \sim 0.5-1 \mathrm{yr}^{-1}$ (Jewitt et al. 2017). While this match at first appears gratifying, it is difficult to quantitatively compare the discoveries of the two interstellar objects given that they have such different physical properties and that they were identified in surveys having very different sky coverage, depth, and cadence parameters. Moreover, the visibility of Q4 is enhanced by a coma, without which the object would likely not have been noticed, whereas 'Oumuamua appeared persistently unresolved.

Based on 'Oumuamua alone the number density of similarly sized interstellar objects was estimated as $N_{1} \sim 0.1 \mathrm{au}^{-3}$ and the differential power-law size distribution as $r_{n}^{-q}$ with $q \geqslant 4$ (Jewitt et al. 2017). Objects larger than 'Oumuamua should accordingly be very rare. The latter was elongated in shape but had an effective radius variously estimated (in order of size) as $r_{O}=45-90 \mathrm{~m}$ (Knight et al. 2017), $\sim 55 \mathrm{~m}$ (Jewitt et al. 2017), 75-79 m (Drahus et al. 2018), $\leqslant 100 \mathrm{~m}$ (Bannister et al. 2017), $102 \mathrm{~m}$ (Meech et al. 2017), and $\leqslant 130 \mathrm{~m}$ (Bolin et al. 2018). For the sake of argument, we take $r_{O}=0.1 \mathrm{~km}$ as the effective radius of 'Oumuamua. Given $q=4$, the cumulative number of interstellar objects larger than radius $r_{n}(\mathrm{~km})$ and inside a sphere of radius $r_{H}(\mathrm{au})$ is just

$$
N\left(r_{n}\right)=\frac{4 \pi r_{H}^{3}}{3} N_{1}\left[\frac{r_{O}}{r_{n}}\right]^{3} .
$$

Q4 was discovered at $r_{H}=3 \mathrm{au}$. Setting $r_{n}=3.8 \mathrm{~km}$ (the upper limit to the radius set by our photometry) in Equation (4), we find $N(3.8)=2 \times 10^{-4}$. We thus consider it very unlikely that Q4 could be as large as our photometric limit allows. In fact, the nucleus is very unlikely to be larger than $1 \mathrm{~km}$ in radius and is most probably just a few hundred meters. For example, Equation (4) gives $N=1$ for $r_{n} \sim 0.2 \mathrm{~km}$ and $N=0.1$ for $r_{n}=0.5 \mathrm{~km}$. A subsequent refinement of our 
number density estimate of 'Oumuamua-sized bodies (from $N_{1}=0.1 \mathrm{au}^{-3}$ to $\sim 0.2 \mathrm{au}^{-3}$ ) by Do et al. (2018) does not materially change this conclusion.

Published upper limits to the number density of interstellar objects fall in the range $N_{1}(1)=10^{-3}$ to $10^{-5} \mathrm{au}^{-3}$, as summarized by Engelhardt et al. (2017). Engelhardt's own best value is $N_{1}(1)<10^{-4} \mathrm{au}^{-3}$, based on involved modeling of three sky surveys each giving zero detections. With $N_{1}(1)<10^{-4} \mathrm{au}^{-3}$, the number of $1 \mathrm{~km}$ radius objects expected within $r_{H}=3$ au of the Sun is $N<10^{-2}$, again showing that the nucleus of Q4 is likely to be a subkilometer body.

Inbound long-period comet $\mathrm{C} / 2019 \mathrm{~J} 2$ recently disintegrated when $1.9 \mathrm{au}$ from the Sun (Jewitt \& Luu 2019), a distance essentially equal to the $q=2.0$ au perihelion distance of Q4. Disintegration is a common yet poorly quantified property of comets, especially those with small nuclei and perihelia (see Sekanina \& Kracht 2018). The nucleus of C/2019 J2, like that of $\mathrm{Q} 4$, had a radius of only a few hundred meters and was probably rotationally disrupted by torques from anisotropic outgassing (Jewitt \& Luu 2019). Continued observations are encouraged to determine whether Q4 might undergo the same fate and, if so, whether it might leave behind a low-activity remnant resembling 'Oumuamua.

\section{Summary}

We present observations of interstellar comet 2I/(2019 Q4) Borisov taken at small solar elongation with the $2.56 \mathrm{~m}$ NOT.

1. The comet is actively emitting dust, as evidenced by a tail of sky-plane length $>1.4 \times 10^{8} \mathrm{~m}$, and by progressive brightening of the coma at about $1 \%$ day $^{-1}$.

2. We infer that strong activity began near heliocentric distance $4.5 \mathrm{au}$ as the comet entered the water ice sublimation zone. The effective particle radius is $\bar{a} \sim 100 \mu \mathrm{m}$, the coma mass $M \sim 1.3 \times 10^{7} \mathrm{~kg}$, and the production rate in dust $d M / d t \sim 2 \mathrm{~kg} \mathrm{~s}^{-1}$.

3. An observational upper limit to the nucleus radius is set at $r_{n}<3.8 \mathrm{~km}$ (albedo $p_{V}=0.1$ assumed). However, based on statistical considerations, we find that the nucleus must be much smaller, and is likely just a few hundred meters in radius.

4. The optical colors $B-V=0.80 \pm 0.05$, $V-R=0.47 \pm 0.03, R-I=0.49 \pm 0.05$, are slightly redder than the Sun, similar to $1 \mathrm{I} /(2017 \mathrm{U1})$ 'Oumuamua and identical within the uncertainties of measurement to the mean color measured for the dust comae of longperiod comets. Like active solar system objects, both interstellar objects lack ultrared matter.

We thank Yoonyoung Kim for comments on the manuscript, David Mkrtichian for a discussion in Kunming, Anlaug Amanda Djupvik for help with the observations, and Thomas Augusteijn for allocating time to this project.

Facility: NOT.

\section{References}

A'Hearn, M. F., Millis, R. C., Schleicher, D. O., et al. 1995, Icar, 118, 223 Bannister, M. T., Schwamb, M. E., Fraser, W. C., et al. 2017, ApJL, 851, L38 Bertini, I., La Forgia, F., Fulle, M., et al. 2019, MNRAS, 482, 2924 Bessell, M. S. 1995, PASP, 107, 672

Blanton, M. R., Bershady, M. A., Abolfathi, B., et al. 2017, AJ, 154, 28

Bohren, C. F., \& Huffman, D. R. 1983, Absorption and Scattering of Light by Small Particles (New York: Wiley)

Bolin, B. T., Weaver, H. A., Fernandez, Y. R., et al. 2018, ApJL, 852, L2 Borisov, G. 2019, MPEC, 2019-R106 (September 11)

Cruikshank, D. P., Roush, T. L., Bartholomew, M. J., et al. 1998, Icar, 135, 389 Dalle Ore, C. M., Barucci, M. A., Emery, J. P., et al. 2015, Icar, 252, 311

Dandy, C. L., Fitzsimmons, A., \& Collander-Brown, S. J. 2003, Icar, 163, 363 Do, A., Tucker, M. A., \& Tonry, J. 2018, ApJL, 855, L10

Drahus, M., Guzik, P., Waniak, W., et al. 2018, NatAs, 2, 407

Engelhardt, T., Jedicke, R., Vereš, P., et al. 2017, AJ, 153, 133

Fitzsimmons, A., Hainaut, O., Meech, K., et al. 2019, ApJL, 885, L9

Guzik, P., Drahus, M., Rusek, K., et al. 2019, NatAs, in press (doi:10.1038/ s41550-019-0931-8)

Holmberg, J., Flynn, C., \& Portinari, L. 2006, MNRAS, 367, 449

Jewitt, D. 1991, in Proc. IAU Coll. 116, Comets in the Post-Halley Era, Astrophysics and Space Science Library, Vol. 167, ed. R. L. Newburn, Jr., M. Neugebauer, \& J. Rahe. (Dordrecht: Kluwer), 19

Jewitt, D. 2015, AJ, 150, 201

Jewitt, D., \& Luu, J. 2019, ApJL, 883, L28

Jewitt, D., Luu, J., Rajagopal, J., et al. 2017, ApJL, 850, L36

Jewitt, D. C. 2002, AJ, 123, 1039

Jewitt, D. C., \& Meech, K. J. 1987, ApJ, 317, 992

Jordi, K., Grebel, E. K., \& Ammon, K. 2006, A\&A, 460, 339

Knight, M. M., Protopapa, S., Kelley, M. S. P., et al. 2017, ApJL, 851, L31 Landolt, A. U. 1992, AJ, 104, 340

Meech, K. J., Weryk, R., Micheli, M., et al. 2017, Natur, 552, 378

Sekanina, Z., \& Kracht, R. 2018, arXiv:1812.07054

Yang, B., Keane, J., Kelley, M., et al. 2019, CBET, 4672

Zubko, E., Videen, G., Shkuratov, Y., \& Hines, D. C. 2017, JQSRT, 202, 104 